\title{
An instability result in the theory of suspension bridges
}

\author{
Clelia Marchionna* — Stefano Panizzi ${ }^{\dagger}$
}

\begin{abstract}
We consider a second order system of two ODE's which arises as a single mode Galerkin projection of the so-called fish-bone [2] model of suspension bridges. The two unknown represent flexural and torsional modes of vibration of the deck of the bridge. The elastic response of the cables is supposed to be asymptotically linear under traction, and asymptotically constant when compressed (a generalization of the slackening regime). We establish a condition depending on a set of 3 parameters under which the flexural motions are unstable provided the energy is sufficiently large.
\end{abstract}

Keywords: suspension bridges, torsional instability, Poincaré map, Hill equation Mathematics Subject Classification: Primary: 37C75; Secondary: 35G31, 34C15

\section{Introduction}

An important issue in the mathematical modeling of suspension bridges is the phenomenon of energy transfer from flexural to torsional modes of vibration along the deck of the bridge. The classical and most accepted explanation for the sudden appearance of large flexural or twisting vibrations is areolastic fluttering, that is a dynamic instability of the elastic structure of the bridge caused by positive feedback between the deflection of the deck of the bridge and the force exerted by the wind.

The purpose of this paper is to provide a contribution to a recent field of research [8] [11] [12] [13] [1] [2] [6] according to which, internal nonlinear resonances, which depend only on the structural properties of the bridge model, may occur even when the aeroelastic coupling is disregarded and, as a consequence, the external forces driven by the wind are not considered. In particular, the main inspiration of this paper comes from the work of F. Gazzola and coworkers [1] [2] [6] to which we refer for more references and motivations.

The suspension bridge model under consideration has been proposed by K.S. Moore [14], revisited and somehow simplified in [2], whose authors called it fish-bone model. It is a two degree of freedom system of PDE's in which the dynamics of the midline of the deck, modeled as an Euler-Bernoulli vibrating beam of length $L$ and width $2 l$, is coupled with the elastic response of the suspension cables acting on the side ends of the deck. The (for example rectangular) cross section of the deck is assumed to be a rigid rod with constant mass density $\rho$, length $2 l$ and negligible thickness with respect to $l$. If we denote by $Y(x, t)$ the vertical downward deflection of the midline

\footnotetext{
* Dipartimento di Matematica del Politecnico, Piazza Leonardo da Vinci 32, 20133 Milano, ITALY
}

${ }^{\dagger}$ Dipartimento di Matematica, Parco Area delle Scienze 53/A, 43125 Parma, ITALY 
of the deck with respect to the unloaded state, and by $\Theta(x, t)$ the angle of rotation of the deck with respect to the horizontal position, the mathematical description of the model is provided by the following system:

$$
\begin{cases}\rho S Y_{t t}+E I Y_{x x x x}+\mathcal{F}(Y+l \sin \Theta)+\mathcal{F}(Y-l \sin \Theta)=0, & 0<x<L, \\ \rho J \Theta_{t t}-G J \Theta_{x x}+l \cos \Theta[\mathcal{F}(Y+l \sin \Theta)-\mathcal{F}(Y-l \sin \Theta)]=0, & 0<x<L,\end{cases}
$$

with the hinged boundary conditions:

$$
Y(0, t)=Y(L, t)=Y_{x x}(0, t)=Y_{x x}(L, t)=0, \quad \Theta(0, t)=\Theta(L, t)=0 .
$$

About the meaning of the constant parameters not yet defined, $S$ is the cross section area, $I$ is the planar second moment of area with respect to the plane $Y=0, J$ is the polar second moment of area with respect to the $x$-axis (being $\rho J$ the torsional moment of inertia of the $x$-section of the bridge) and $E$ and $G$ are respectively Young's modulus and the shear modulus (being EI the flexural rigidity and $G J$ the torsional rigidity). The force $\mathcal{F}$ represents the restoring action exerted by the hangers in addition to gravity, and is applied to both extremities of the deck whose displacements from the unloaded state are given by $Y \pm l \sin \Theta$.

A reasonable choice (see [2], [14]), is to assume that the suspension cables do not resist compression, and behave as linear springs of elastic constant $\mathrm{k}>0$ if stretched (here $g$ is the gravity). In this case we have the following nonlinear response (slackening regime):

$$
\mathcal{F}(r)=\mathrm{k}\left[\left(r+r_{0}\right)^{+}-r_{0}\right], \quad r_{0}=\rho S g / 2 \mathrm{k}
$$

Now, as usual, we make a first simplification on this model: we denote by $Z(x, t)$ the vertical displacement with respect to the midline of the right edge of the road at position $x$, and $f(r)=$ $\frac{1}{\rho S} \mathcal{F}(r)$; if we assume that, at least at the beginning, $\Theta$ is small enough, we have $\sin \Theta \sim \Theta$, $\cos \Theta \sim 1$. Setting $Z=l \Theta$, the system (1) becomes

$$
\begin{cases}Y_{t t}+\frac{E I}{\rho S} Y_{x x x x}+f(Y+Z)+f(Y-Z)=0, & 0<x<L, \quad t>0 \\ Z_{t t}-\frac{G}{\rho} Z_{x x}+\frac{S l^{2}}{J}(f(Y+Z)-f(Y-Z))=0, & 0<x<L, \quad t>0\end{cases}
$$

with the hinged boundary conditions:

$$
Y(0, t)=Y(L, t)=Y_{x x}(0, t)=Y_{x x}(L, t)=0, \quad Z(0, t)=Z(L, t)=0 .
$$

Actually the results that we present in this paper depend only on certain properties of the non linear function $f$, essentially the fact that it has a finite limit slope as the displacements at the

side edges of the deck $Y \pm Z$ get large. For this reason, we slightly generalize the law (3), with the following:

\section{Assumption (H)}

a) $f$ is an increasing, continuous function such that $f(0)=0$;

b) $f$ is piecewise $C^{1}$, that is it has continuous derivative with the exception of a finite (eventually empty) set of points $r_{1}<r_{2}<\ldots<r_{n}$ not including zero in which there exist the finite limits:

$$
\lim _{r \rightarrow r_{i}^{ \pm}} f^{\prime}(r) ;
$$


c) $f$ has asymptotically constant slope as $r \rightarrow \infty$, more precisely there exist the limits:

$$
\lim _{r \rightarrow+\infty} f^{\prime}(r)=m>0, \quad \lim _{r \rightarrow-\infty} f^{\prime}(r)=0 .
$$

We recall that under assumption (H) both problems (4)-(5) and (1)-(2), when supplemented with sufficiently regular initial data, are well-posed in suitable Sobolev spaces (see [2]). In addition they have two immediate but crucial properties: they are conservative, i.e. the total energy does not change as the system evolves over time; they admit what we call pure flexural solutions, that is motions in which the cross sections of the deck remain horizontal at all times, and of course no torsional vibration may occur. Moreover the pure flexural solutions are the same for both models.

We will refer in this paper mostly to the "pre-linearized" system (4) for statements and examples, but the analytical results hold also for the system (1) (see the end of section 4).

The problem we want to address in this note, as it has been extensively introduced and motivated in [2], may be summarized as follows: starting from a slightly perturbed pure flexural motion, is it possible that, due to the nonlinearity of the model, a relevant part of the energy may be transferred to a torsional mode of vibration? In case of affirmative answer, it amounts to say that, at least for some values of the parameters, the pure flexural motions are unstable. Therefore it arises the problem of finding simple conditions to characterize the instability domains.

A first interesting investigation could be studying the interaction between a single flexural mode and another single torsional mode. In this paper we choose to start with the two first modes.

Precisely, after a suitable rescaling of the space variable, we assume that the displacements are well approximated by their first mode of vibration. This means that, owing to the boundary conditions, we have that

$$
Y(x, t) \simeq y(t) \sin x, \quad Z(x, t) \simeq z(t) \sin x, \quad 0 \leq x \leq \pi .
$$

Then, through a Galerkin projection, we reduce the system (4) to a coupled system of two nonlinear second order ODE's:

$$
\begin{aligned}
& \ddot{y}(t)+\alpha y(t)+\tilde{f}(y(t)+z(t))+\tilde{f}(y(t)-z(t))=0, \\
& \ddot{z}(t)+\beta z(t)+\gamma[\tilde{f}(y(t)+z(t))-\tilde{f}(y(t)-z(t))]=0,
\end{aligned}
$$

where $\alpha=\frac{E I \pi^{4}}{\rho S L^{4}}, \beta=\frac{G \pi^{2}}{\rho L^{2}}$ and $\gamma=\frac{S l^{2}}{J}$. The function $\tilde{f}$ takes into account the projection onto the first eigenmode, and it is given by the following transformation formula (see Section 2 for its properties),

$$
\tilde{f}(r)=\frac{2}{\pi} \int_{0}^{\pi} f(r \sin x) \sin x d x
$$

We note that in the forthcoming computations, owing to the symmetry of $\sin x$, we rewrite the above integral on $[0, \pi]$ as twice the integral on $[0, \pi / 2]$, i.e.

$$
\tilde{f}(r)=\frac{4}{\pi} \int_{0}^{\pi / 2} f(r \sin x) \sin x d x
$$


The reduced system (6)-(7) forms a Hamiltonian system whose nonnegative conserved energy $\mathcal{E}$ as a function of the 4 phase space variables, is given by

$$
\mathcal{E}(y, \dot{y}, z, \dot{z})=\frac{\dot{y}^{2}}{2}+\frac{\dot{z}^{2}}{2 \gamma}+\frac{\alpha}{2} y^{2}+\frac{\beta}{2 \gamma} z^{2}+\tilde{F}(y+z)+\tilde{F}(y-z),
$$

where $\tilde{F}(r)=\int_{0}^{r} \tilde{f}(s) d s$.

As an immediate consequence of the conservative character of the system (6)-(7), all solutions are bounded and globally defined on $(-\infty, \infty)$. In addition to this, the ODE's system (6)-(7) admits pure flexural periodic solutions $(w(t), 0)$ as well, provided that $w(t)$ solves the following Duffing-type equation,

$$
\ddot{w}(t)+\alpha w(t)+2 \tilde{f}(w(t))=0,
$$

In this note we shall prove that, if the energy of the system is sufficiently large and the parameters satisfy a certain condition, see (11) below, every pure flexural periodic solution is unstable. To simplify notations, we introduce the three factors involved in characterizing regions of instability:

$$
\phi_{0}:=\sqrt{\frac{\beta+2 \gamma m}{\alpha+2 m}} \pi, \quad \phi_{1}:=\sqrt{\frac{\beta}{\alpha}} \pi, \quad q=\sqrt{\frac{\beta+2 \gamma m}{\beta}} .
$$

Our main result is the following:

Theorem 1 Under the assumption $(\boldsymbol{H})$, assume that the following condition holds true,

$$
\left|\cos \phi_{0} \cos \phi_{1}-\frac{q+q^{-1}}{2} \sin \phi_{0} \sin \phi_{1}\right|>1 .
$$

Then there exists an energy level $\mathcal{E}_{0}$ such that, if $\mathcal{E}(w(0), \dot{w}(0), 0,0)>\mathcal{E}_{0}$, the pure flexural periodic solution $w(t)$ of the equation (9) is unstable.

In order to illustrate the meaning and the relevance of condition (11) in our main theorem, in section 3 we provide several numerical examples involving different slacking functions.

The significance of instability in Theorem 1 is the same presented by Cazenave \& Weissler in [3] for the study of two modes solutions of the Kirchhoff nonlinear string: there exists a two-dimensional submanifold $\mathcal{M}$ of the (three-dimensional) fixed energy level manifold $\mathcal{E}(y, \dot{y}, z, \dot{z})=\mathcal{E}_{k}>\mathcal{E}_{0}$ with the following property: if $y, z$ are solutions of (6)-(7) with initial data $\left(y_{0}, y_{1}, z_{0}, z_{1}\right) \in \mathcal{M}$, then $z$ and $\dot{z}$ converge exponentially to 0 as $t \rightarrow+\infty$ and there exists a solution $w$ of the equation (9) with energy $\mathcal{E}_{k}$, such that $y-w$ and $\dot{y}-\dot{w}$ converge exponentially to 0 as $t \rightarrow+\infty$. Since the system (6)-(7) is time reversible, this result also proves the existence of a non trivial unstable manifold for the pure flexural solution $w$.

Theoretical and numerical investigations of torsional instabilities for finite dimensional projections of the suspension bridge model have been extensively carried out by the authors of [2], in which a cubic nonlinear response of the cables is considered:

$$
f(r)=r+\gamma r^{3} .
$$

Of course, such case is not covered by our assumption $(\mathbf{H})$. However, we believe that our work has some interesting aspects: it covers the case of the slackening regime, and it provides a simple characterization of instability regions. For example, it seems unlikely to have some kind of instability 
at high energies when $\alpha=\beta$ or $\sqrt{\beta / \alpha}$ is an integer $\left(\sin \phi_{1}=0\right)$; anyway that occurrence does not necessarily happen in actual bridges (see the remark below).

The mathematical side of the present work has been mainly influenced by the aforementioned paper [3] (see also [7]). In fact, after reduction of the system to fixed energy levels $\mathcal{E}_{k}$ and linearization around the corresponding periodic flexural solution (Section 4), we borrowed the idea of studying the asymptotic linearized system as $\mathcal{E}_{k} \rightarrow+\infty$ (Section 5). In our case, this system turns out to be simple to be studied, allowing us to provide a precise condition for instability. Unfortunately, the method does not give any estimate on the size of the critical energy level $\mathcal{E}_{0}$ (flutter energy), where the instability begins to occur.

Remark 1 Let us discuss the dependence of the parameters on the structural properties of the bridge and the hangers: First we observe that, if the behaviour of the hangers is described by (3) the slope $m$ depends on the elastic behavior of the hangers (the elasticity constant $\mathrm{k}$ ) and on the structural properties of the bridge; precisely $m=\frac{\mathrm{k}}{\rho S}$, where the cross section area $S$ depends on the width and the thickness of the bridge. Let us assume that the section of the bridge is a rectangle of width $2 l$ and height $h$ : obviously we have $S=2 l h, m=\frac{\mathrm{k}}{2 \rho l h}$. The two momenta of area (flexural and torsional) become $I=\frac{l h^{3}}{6}$, and $J=\frac{2}{3} l^{3} h+\frac{l h^{3}}{6} \sim \frac{2}{3} l^{3} h$ if $h<<l$. Finally we have $\alpha=\frac{\pi^{4}}{L^{4}} \frac{E h^{2}}{12 \rho}$, $\beta=\frac{\pi^{2}}{L^{2}} \frac{G}{\rho}, \gamma \sim 3$. The crucial dependence on the width of the bridge $2 l$ and the rigidity of the hangers $\mathrm{k}$ is hidden in the parameter $m$.

\section{A few properties of the function $\tilde{f}$}

Since, from now on, only the properties of the transformed function $\tilde{f}$ matter, in this section we briefly resume what we shall need in the rest of the paper. With the aid of the following proposition we shall replace condition $(\mathbf{H})$ with the handier condition $(\tilde{\mathbf{H}})$.

Proposition 2.1 Under the assumption $(\boldsymbol{H})$ on the function $f$, the transformed function $\tilde{f}$ as defined in (8), satisfies the following

\section{Condition $(\tilde{\mathbf{H}})$}

a) $\tilde{f}$ is an increasing, $C^{1}(\mathbb{R})$ function such that $\tilde{f}(0)=0$;

$\tilde{b})$ there exist the same limits as in c) of assumption $(\boldsymbol{H})$ :

$$
\lim _{r \rightarrow+\infty} \tilde{f}^{\prime}(r)=m>0, \quad \lim _{r \rightarrow-\infty} \tilde{f}^{\prime}(r)=0 .
$$

PROOF. We shall show that $\tilde{f} \in C^{1}(\mathbb{R})$ with

$$
\tilde{f}^{\prime}(r)=\frac{4}{\pi} \int_{0}^{\pi / 2} f^{\prime}(r \sin x) \sin ^{2} x d x, \quad r \in \mathbb{R} .
$$

To see this, assume for simplicity, that there exists a unique point $r_{1}>0$ as in condition $\left.\mathrm{b}\right)$. Then $g(r, x)=f(r \sin x) \sin x$ is of class $C^{1}$ in $\left(-\infty, r_{1}\right] \times[0, \pi / 2]$, and the usual derivative under integral sign rule applies, which yields formula (12). In the case when $r>r_{1}$, we may write

$$
\tilde{f}(r)=\frac{4}{\pi} \int_{0}^{x_{1}(r)} g(r, x) d x+\frac{4}{\pi} \int_{x_{1}(r)}^{\pi / 2} g(r, x) d x,
$$


in which $x_{1}(r)=\arcsin \left(r_{1} / r\right)$ is a $C^{1}$ function in a neighborhood $U$ of any given $\bar{r}>r_{1}$, and $g$ is of class $C^{1}$ both in $\left\{(r, x): r \in U, 0 \leq x \leq x_{1}(r)\right\}$, and in $\left\{(r, x): r \in U, x_{1}(r) \leq x \leq \pi / 2\right\}$. After differentiation with respect to $r$, the terms containing $x_{1}^{\prime}(r)$ cancel out, and formula (12) is again satisfied. The general case is harder to write down but it follows by the same argument. Note that the limits in $\tilde{b}$ ) follow easily from (12), the dominated convergence theorem and the fact that $4 / \pi \int_{0}^{\pi / 2} \sin ^{2} x d x=1$.

Incidentally we observe that the transform $f \mapsto \tilde{f}$ falls within the family of Abel transforms [4]. To see this, after the substitution $t=r \sin x$ in the integral defining $\tilde{f}$, we get

$$
\tilde{f}(r)=\frac{4}{\pi} \int_{0}^{r} \frac{t f(t)}{\sqrt{r^{2}-t^{2}}} d t .
$$

There also exists an inversion formula which holds true for sufficiently smooth functions:

$$
f(r)=\frac{1}{2 r} \frac{d}{d r} \int_{0}^{r} \frac{t \tilde{f}(t)}{\sqrt{r^{2}-t^{2}}} d t .
$$

Remark 2 If the forcing term is defined by (3), that is

$$
f(r)=m\left[\left(r+r_{0}\right)^{+}-r_{0}\right], \quad r_{0}=g / 2 m,
$$

the function $\tilde{f}$ is easy to compute, and precisely we have

$$
\tilde{f}(r)=m r, \quad r \geq-r_{0}, \quad \tilde{f}(r)=-\frac{g}{\pi}\left[\frac{r}{r_{0}} \arcsin \left(\frac{r_{0}}{r}\right)+\sqrt{1-\left(\frac{r_{0}}{r}\right)^{2}}\right], \quad r \leq-r_{0} .
$$

\section{$3 \quad$ Numerical examples}

In this section we report a few numerical examples in which a couple of functions $\tilde{f}$ satisfying the Condition $(\tilde{\mathbf{H}})$ are considered. All the simulations have been performed with the standard routine MATLAB ODE45. To keep track of the numerical accuracy, we have evaluated the time evolution of the (conserved) total energy: its variation on a time interval $[0,200]$ is around $2 \%, 3.5 \%$ depending on its effective size.

First we show an example with a function $\tilde{f}$ like the one presented in (13), which corresponds to the simple model of slackening presented in the introduction:

$$
f(r)=m r, \quad r \geq-r_{0}, \quad f(r)=-h, \quad r \leq-r_{0}, \quad r_{0}=\frac{m}{h}
$$

Precisely, for the transformed function $\tilde{f}$, we have

$$
\tilde{f}(r)=m r, \quad r \geq-r_{0}, \quad \tilde{f}(r)=-\frac{2}{\pi} h\left[\frac{r}{r_{0}} \arcsin \left(\frac{r_{0}}{r}\right)+\sqrt{1-\left(\frac{r_{0}}{r}\right)^{2}}\right], \quad r \leq-r_{0} .
$$

We have kept fixed the three parameters in the system (6)-(7): $\alpha=1, \beta=2, \gamma=3$ and set $m=h=1$. In this case the absolute value of the instability discriminant $\Delta=\cos \phi_{0} \cos \phi_{1}-(q+$ 

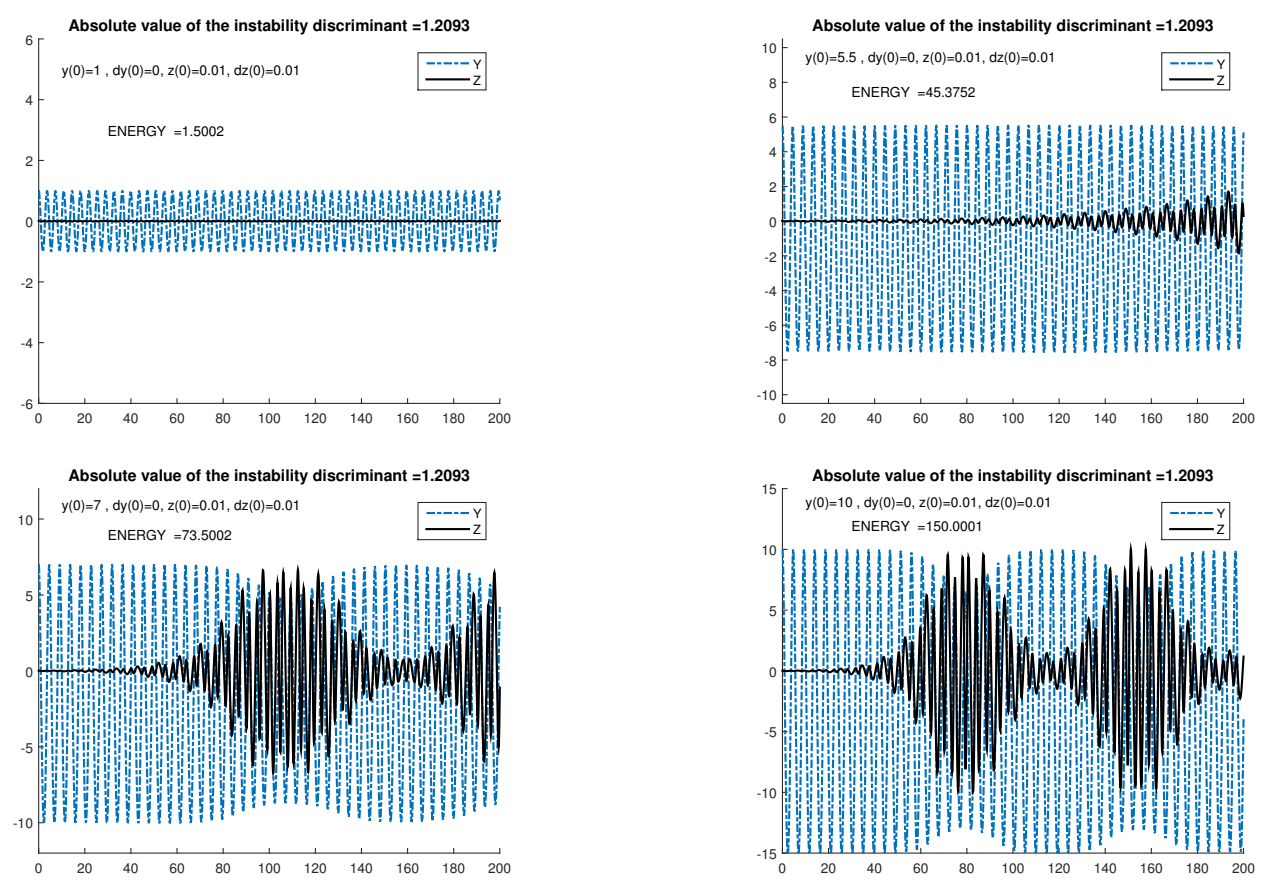

Figure 1: First example: evolution of instability as the energy increases. The parameters are fixed: $\alpha=1, \beta=2, \gamma=3, m=h=1$. The absolute value of the instability discriminant is 1.209.

$\left.q^{-1}\right) / 2 \sin \phi_{0} \sin \phi_{1}$ is 1.209 , so that our result yields instability of the pure torsional solution for sufficiently high energy. In Figure 1 we show the simulations when $\dot{y}(0)=0, z(0)=\dot{z}(0)=0.01$, and the initial value of $y$ is increased from 1 to 10. It is evident the emergence of instability in the fixed time interval as the energy increases.

Then we present another example in which the function $\tilde{f}$, depending on the variable asymptotic slope $m$ at $+\infty$, still satisfies the Condition $(\tilde{\mathbf{H}})$, but is not asymptotic to any straight line as $|r| \rightarrow \infty:$

$$
\tilde{f}(r)=\frac{m}{2}\left(x+\sqrt{x^{2}+1}-1\right)+\frac{x^{5 / 3}}{x^{4 / 3}+1} .
$$

We have kept fixed the 4 parameters in the system (6)-(7): $\alpha=1, \beta=3, \gamma=3$, and $m=5$ so that the instability discriminant $\Delta=1.7959$. Progression to the transfer of energy between the two modes is very similar to the first example (see Figure 2).

On the contrary, if we set $\alpha=\beta$, our sufficient condition for instability at high energies doesn't hold. Actually we can see in Figure 3, that $z, \dot{z}$ remain small, despite the quite high energy with respect to the previous examples. Both examples of functions $\tilde{f}$ are reported. From the numerical point of view, we note that in this case, during time evolution, the variation in percentage of the energy is less than $0.2 \%$. 

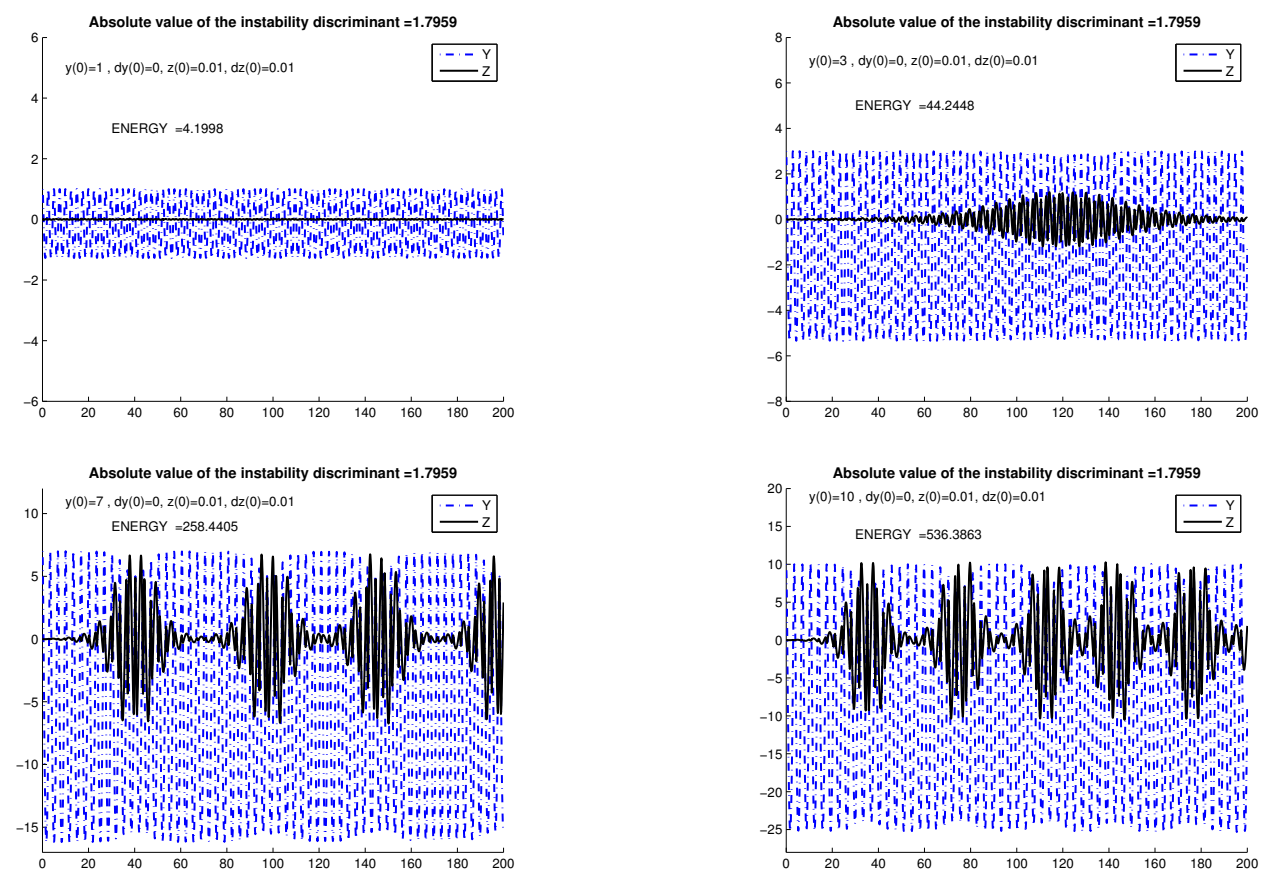

Figure 2: Second example: emergence of instability as the energy increases. The parameters are fixed: $\alpha=1, \beta=2, \gamma=3, m=5$. The absolute value of the instability discriminant is 1.7959 .
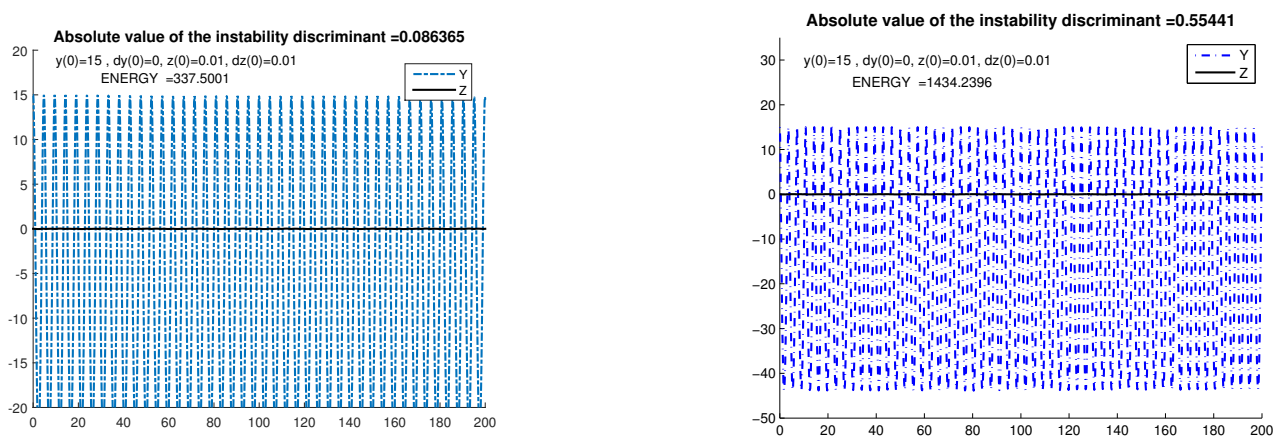

Figure 3: $\alpha=\beta$, the modulus of the instability discriminant is always $<1$. Stability for both examples: on the left the first example, on the right the second one 


\section{The Poincaré map and its linearization}

In this section we construct the Poincaré map around a pure flexural periodic solution of (6)-(7). In order to reduce by 1 the degrees of freedom, we shall consider orbits constrained to a fixed energy level. As a consequence, the instability characterization of the periodic orbit reduces to the value of a single parameter as we show in Proposition 4.1. The discussion of this section should be considered more or less classical. However having not found a reference which outlines precisely the needed arguments, we reported the proof of Proposition 4.1.

Let us fix a positive number $k>0$, and consider the pure flexural periodic solution $w_{k}$ solving the problem,

$$
\ddot{w}_{k}(t)+\alpha w_{k}(t)+2 \tilde{f}\left(w_{k}(t)\right)=0, \quad w_{k}(0)=k, \quad \dot{w}_{k}(0)=0,
$$

whose invariant total energy is given by,

$$
\mathcal{E}_{k}:=\mathcal{E}(k, 0,0,0)=\alpha \frac{k^{2}}{2}+2 \tilde{F}(k) .
$$

Let us denote by $\tau_{k}$ its period, and by

$$
\Gamma_{k}=\left\{\left(w_{k}(t), \dot{w}_{k}(t), 0,0\right): t \in \mathbb{R}\right\}
$$

its orbit in the 4-dimensional phase space. Referring to the system (6)-(7), on the invariant 3dimensional energy level $\left\{\mathcal{E}=\mathcal{E}_{k}\right\}$, we shall consider a neighborhood of the origin on the 2dimensional section $\{\dot{y}=0\}$, parametrized by the 'torsional' components $(z, \dot{z})$. More precisely, let $\bar{B}_{\delta}$ be the closed disk around $(0,0)$ of radius $\delta>0$. The radius $\delta$ will be chosen according to several smallness conditions, the first of these conditions is that

$$
\bar{B}_{\delta} \subset\left\{\left(z_{0}, z_{1}\right) \in \mathbb{R}^{2}: \mathcal{E}\left(0,0, z_{0}, z_{1}\right)<\mathcal{E}_{k}\right\} .
$$

We next consider the function $G\left(y, z_{0}, z_{1}\right)=\mathcal{E}\left(y, 0, z_{0}, z_{1}\right)$, of class $C^{2}$ with respect to all arguments. We note that $G(k, 0,0)=\mathcal{E}_{k}$, and that $\partial G / \partial y(k, 0,0)=\alpha k+2 \tilde{f}(k)>0$, so that by the implicit function theorem, if $\delta$ is sufficiently small, there exists a $C^{2}$ function $\eta: \bar{B}_{\delta} \longrightarrow(0,+\infty)$, such that for every $\left(z_{0}, z_{1}\right) \in \bar{B}_{\delta}$,

$$
\eta(0,0)=k, \quad \mathcal{E}\left(\eta\left(z_{0}, z_{1}\right), 0, z_{0}, z_{1}\right)=\mathcal{E}_{k} .
$$

Now for any given $\left(z_{0}, z_{1}\right) \in \bar{B}_{\delta}$, let $\left(y\left(t ; z_{0}, z_{1}\right), z\left(t ; z_{0}, z_{1}\right)\right)$ be the solution of the system (6)-(7), corresponding to the initial data,

$$
y(0)=\eta\left(z_{0}, z_{1}\right), \quad \dot{y}(0)=0, \quad z(0)=z_{0}, \quad \dot{z}(0)=z_{1} .
$$

Again, by choosing $\delta$ small enough, thanks to the $C^{1}$-dependence on the initial data, and by a classical implicit function argument (see e.g [10] p. 243), there exits the first positive time of return $T=T\left(z_{0}, z_{1}\right)$, implicitly defined as the first time in which the solution crosses the section $\{\dot{y}=0, y>0\}$, that is

$$
y\left(T ; z_{0}, z_{1}\right)>0, \quad \dot{y}\left(T ; z_{1}, z_{1}\right)=0 .
$$

Clearly since $\partial \dot{y} / \partial t\left(\tau_{k} ; 0,0\right)=-\alpha k-2 \tilde{f}(k) \neq 0$, it follows by the previous choice of the radius $\delta$, that the dependence of $T$ on $\left(z_{0}, z_{1}\right)$ is of class $C^{1}\left(\bar{B}_{\delta}\right)$. 
Finally we can now define the (iso-energetic) first return map or Poincaré map $\mathcal{P}_{k}$ relative to the periodic orbit $\Gamma_{k}$, as the following $C^{1}$ map:

$$
\mathcal{P}_{k}: \bar{B}_{\delta} \longrightarrow \mathbb{R}^{2}, \quad \mathcal{P}_{k}\left(z_{0}, z_{1}\right)=\left(z\left(T\left(z_{0}, z_{1}\right) ; z_{0}, z_{1}\right), \dot{z}\left(T\left(z_{0}, z_{1}\right) ; z_{0}, z_{1}\right)\right) .
$$

It is well-known that the stability properties of the flow around the closed orbit $\Gamma_{k}$ are closely connected to those of the discrete dynamical system generated by the map $\mathcal{P}_{k}$ around its fixed point $(0,0)$. In particular we have the following

Proposition 4.1 Let $\mathcal{L}_{k}=D \mathcal{P}_{k}(0,0)$ be the differential of the Poincaré map at the origin, which we shall write in matrix form as follows

$$
\mathcal{L}_{k}=\left(\begin{array}{ll}
L_{11} & L_{12} \\
L_{21} & L_{22}
\end{array}\right)
$$

Then we have the following properties:

A) $\operatorname{det} \mathcal{L}_{k}=1$ and $L_{11}=L_{22}$

B) If $\left|L_{11}\right|>1$, then $\mathcal{L}_{k}$ has two real eigenvalues $\lambda_{1}, \lambda_{2}$, such that $\left|\lambda_{1}\right|>1, \lambda_{2}=1 / \lambda_{1}$. In this case the periodic orbit $\Gamma_{k}$ is unstable.

We refer to the Introduction (after Theorem 1) for the significance of instability in part B) of the previous Proposition.

The concrete characterization of the differential $\mathcal{L}_{k}$ is standard in the case when the Poincarè map is not constrained to a fixed energy level, in our case the construction is very similar, and it can be found in full details in [3]. First we linearize the equation (7) around the periodic orbit $\Gamma_{k}$ by considering the Hill equation,

$$
\ddot{u}(t)+\left(\beta+2 \gamma \tilde{f}^{\prime}\left(w_{k}(t)\right)\right) u(t)=0,
$$

with initial data $(u(0), \dot{u}(0))=(a, b) \in R^{2}$. Then $\mathcal{L}_{k}$ is defined as follows,

$$
\mathcal{L}_{k}(a, b)=\left(u\left(\tau_{k}\right), \dot{u}\left(\tau_{k}\right)\right),
$$

in which, we recall, $\tau_{k}$ is the period of $w_{k}(t)$.

PROOF OF PROPOSITION 4.1 (PART A). Let $u^{0}(t)$ and $u^{1}(t)$ be the two solutions of (17) corresponding to the initial conditions $(1,0)$ e $(0,1)$ respectively. Then $\mathcal{L}_{k}$ is the fundamental matrix evaluated at time $\tau_{k}$ :

$$
\left(\begin{array}{ll}
L_{11} & L_{12} \\
L_{21} & L_{22}
\end{array}\right)=\left(\begin{array}{ll}
u^{0}\left(\tau_{k}\right) & u^{1}\left(\tau_{k}\right) \\
\dot{u}^{0}\left(\tau_{k}\right) & \dot{u}^{1}\left(\tau_{k}\right)
\end{array}\right)
$$

From Lioville's formula on the determinant of the fundamental matrix, we have that $\operatorname{det} \mathcal{L}_{k}=1$.

Next we remark that, thanks to time reversibility and uniqueness of the Cauchy problem (14), $w(\cdot)$ is even. Thus $w_{k}\left(\tau_{k}-t\right)=w_{k}(-t)=w_{k}(t)$. It follows that whenever $u(t)$ is a solution of the equation (17), also the function $\bar{u}(t)=u\left(\tau_{k}-t\right)$ solves the same equation. In particular, since 
$\bar{u}^{0}(0)=u^{0}\left(\tau_{k}\right)=L_{11}, \dot{\bar{u}}^{0}(0)=-\dot{u}^{0}\left(\tau_{k}\right)=-L_{21}$, and $\bar{u}^{0}\left(\tau_{k}\right)=u^{0}(0)=1, \dot{\bar{u}}^{0}\left(\tau_{k}\right)=-\dot{u}^{0}(0)=0$, we have that

$$
\left(\begin{array}{ll}
L_{11} & L_{12} \\
L_{21} & L_{22}
\end{array}\right)\left(\begin{array}{c}
L_{11} \\
-L_{21}
\end{array}\right)=\left(\begin{array}{l}
1 \\
0
\end{array}\right)
$$

Analogously, by applying the same argument to $u^{1}(t)$ and $\bar{u}_{1}(t)$, we have that

$$
\left(\begin{array}{ll}
L_{11} & L_{12} \\
L_{21} & L_{22}
\end{array}\right)\left(\begin{array}{c}
L_{12} \\
-L_{22}
\end{array}\right)=\left(\begin{array}{c}
0 \\
-1
\end{array}\right)
$$

Therefore we obtain the following relations,

$$
L_{22}^{2}-L_{12} L_{21}=L_{11}^{2}-L_{12} L_{21}=1, \quad L_{21}\left(L_{11}-L_{22}\right)=L_{12}\left(L_{11}-L_{22}\right)=0,
$$

which together with $\operatorname{det} \mathcal{L}_{k}=1$ yield $L_{11}=L_{22}$.

Let's set $a=L_{11}=u^{0}\left(\tau_{k}\right)$ : then the eigenvalues of $\mathcal{L}_{k}$ are $\lambda_{1,2}=a \pm \sqrt{a^{2}-1}$. If $|a|>1$, they are both real, simple, one the inverse of the other, so the origin is unstable for the map $\mathcal{L}_{k}$. (On the contrary is easy to verify that $(0,0)$ is stable for $\mathcal{L}_{k}$ if $\left.|a| \leq 1\right)$.

(PART B) Let us consider the linear map $\mathcal{L}_{k}$ under the assumption that there are two real eigenvalues $\lambda$ and $1 / \lambda$, with $|\lambda|<1$. Owing to a classical result (see for example [9], chapter IX), if $\delta$ is small enough, there exists a regular curve $\mathcal{C}$ passing through $(0,0)$, which is invariant for $\mathcal{P}_{k}$, and such that

$$
\left|\mathcal{P}_{k}\left(z_{0}, z_{1}\right)\right| \leq \nu\left|\left(z_{0}, z_{1}\right)\right|, \quad \nu<1 \quad \forall\left(z_{0}, z_{1}\right) \in \mathcal{C} \cap \bar{B}_{\delta},
$$

so that we clearly have $\mathcal{P}_{k}^{n}\left(z_{0}, z_{1}\right) \in \mathcal{C} \cap \bar{B}_{\delta}$, and $\left|\mathcal{P}_{k}^{n}\left(z_{0}, z_{1}\right)\right| \leq \nu^{n}\left|\left(z_{0}, z_{1}\right)\right|$, for every $n \geq 1$. From now on, we shall consider solutions $y=y\left(t ; z_{0}, z_{1}\right), z=z\left(t ; z_{0}, z_{1}\right)$ of the system (6)-(7) corresponding to initial data $\left(\eta\left(z_{0}, z_{1}\right), 0, z_{0}, z_{1}\right)$, in which $\left(z_{0}, z_{1}\right) \in \mathcal{C} \cap \bar{B}_{\delta}$, and $\eta\left(z_{0}, z_{1}\right)$ is defined by (15).

First we define the sequence $t_{n}$ of the n-times of return of $y(t)$ to the section $\{y>0, \dot{y}=0\}$, which means that for every $n \in \mathbb{N}$ :

$$
\dot{y}\left(t_{n}\right)=0, \quad y\left(t_{n}\right)>0 .
$$

If we set $\mu=\max _{\bar{B}_{\delta}} T\left(z_{0}, z_{1}\right)$, where $T\left(z_{0}, z_{1}\right)$ is the first return time as defined in (16), we obviously have $t_{0}=0, t_{1}=T, t_{n+1}-t_{n} \leq \mu$. Moreover $\left(z^{2}\left(t_{n}\right)+\dot{z}^{2}\left(t_{n}\right)\right) \leq \delta^{2} \nu^{2 n}$.

Since the function $z(t)$, and its first and second derivatives are bounded (the vector field defined by the system (6)-(7) is bounded on a fixed energy level), we obtain that

$$
z^{2}(t)+\dot{z}^{2}(t) \leq C\left(z^{2}\left(t_{n}\right)+\dot{z}^{2}\left(t_{n}\right)\right) \quad \forall t \in\left[t_{n}, t_{n+1}\right] \subseteq\left[t_{n}, t_{n}+\mu\right],
$$

where $C$ is a suitable constant depending on $\tilde{f}, k$ and $\mu$ but not on $n$. (We will always use the letter $C$ for every constant, even if it is not the same in different lines)

Putting together the previous estimates, we get that,

$$
z^{2}(t)+\dot{z}^{2}(t) \leq C \nu^{2 n} \quad \forall t \in\left[t_{n}, t_{n+1}\right] .
$$

To convert the previous inequality to a continuos in time exponential decay, we introduce the number $\epsilon>0$ such that $e^{-\epsilon \mu}=\nu$. Since $t_{n} \leq n \mu$, for $t \in\left[t_{n}, t_{n+1}\right]$ we have

$$
e^{-2 \epsilon t} \geq e^{-2 \epsilon t_{n+1}} \geq e^{-2 \epsilon(n+1) \mu}=e^{-2 \epsilon \mu} \nu^{2 n} \text {. }
$$


Hence we conclude the first step of our proof:

$$
z^{2}(t)+\dot{z}^{2}(t) \leq C e^{-2 \epsilon t}, \quad \forall t>0 .
$$

Let us now consider the energy evaluated at the time $t_{n}$ : taking into account (19), we note that,

$$
\mathcal{E}_{k}=\mathcal{E}\left(y\left(t_{n}\right), 0, z\left(t_{n}\right), \dot{z}\left(t_{n}\right)\right), \quad\left(z\left(t_{n}\right), \dot{z}\left(t_{n}\right)\right) \in \bar{B}_{\delta},
$$

so that by definition (15), $y\left(t_{n}\right)=\eta\left(z\left(t_{n}\right), \dot{z}\left(t_{n}\right)\right)$. It follows that the following inequality holds true, for every $n \in \mathbb{N}$,

$$
\left|y\left(t_{n}\right)-w_{k}\left(n \tau_{k}\right)\right|=\left|y\left(t_{n}\right)-k\right| \leq \max _{(a, b) \in \bar{B}_{\delta}}|\nabla \eta(a, b)|\left|\left(z\left(t_{n}\right), \dot{z}\left(t_{n}\right)\right)\right| \leq C e^{-\epsilon t_{n}} .
$$

As we did for the estimate of $z(t)$, we can prove that

$$
\left\|y\left(t_{n}+\cdot\right)-w(\cdot)\right\|_{C^{1}([0, \mu])} \leq C e^{-\epsilon t_{n}},
$$

for every $n \geq 1$.

Next we claim that, for every $n \in \mathbb{N}$,

$$
\left|t_{n+1}-t_{n}-\tau_{k}\right| \leq C e^{-\epsilon t_{n}} .
$$

Indeed this follows immediately by the mean value theorem, and by the inequality (20), if we remark that $t_{n+1}-t_{n}=T\left(z\left(t_{n}\right), \dot{z}\left(t_{n}\right)\right)$, and that $\tau_{k}=T(0,0)$.

In particular from (22) we get that, at least for $n \geq n_{0}$, that $t_{n+1}-t_{n}>\tau_{k} / 2$ and that $t_{n}>n c$ for a suitable positive constant $c$. Moreover, by using a classical argument, we can prove that exist a phase $\theta$, such that:

$$
\left|t_{n}-n \tau_{k}-\theta\right| \leq C e^{-\epsilon t_{n}}
$$

In fact the phase is defined as the sum of the following series, $\theta=: \sum_{j=0}^{+\infty}\left(t_{j+1}-t_{j}-\tau_{k}\right)$ which, thanks to (22) is convergent. We have $t_{n}-n \tau_{k}=\sum_{j=0}^{n-1}\left(t_{j+1}-t_{j}-\tau_{k}\right)$ and

$$
\left|t_{n}-n \tau_{k}-\theta\right|=\left|\sum_{j=n}^{\infty}\left(t_{j+1}-t_{j}-\tau_{k}\right)\right| \leq C \sum_{j=n}^{\infty} e^{-\epsilon t_{j}} \leq C e^{-\epsilon t_{n}} \sum_{j=n}^{\infty} e^{-\epsilon\left(t_{j}-t_{n}\right)} \leq C e^{-\epsilon t_{n}} \sum_{j=0}^{\infty} e^{-\epsilon c j} .
$$

By the inequality (23), and thanks to the boundedness in norm $C^{2}$ of the periodic solution $w_{k}$, we can conclude that

$$
\left\|w_{k}\left(n \tau_{k}+\cdot\right)-w_{k}\left(t_{n}-\theta+\cdot\right)\right\|_{C^{1}[0, \mu]} \leq C e^{-\epsilon t_{n}} .
$$

Putting together this last estimate and (21), we have

$$
\left\|y\left(t_{n}+\cdot\right)-w_{k}(\cdot)+w_{k}\left(n \tau_{k}+\cdot\right)-w_{k}\left(t_{n}-\theta+\cdot\right)\right\|_{C^{1}([0, \mu])} \leq C e^{-\epsilon t_{n}} \leq C e^{\epsilon \mu} e^{-\epsilon t_{n+1}} .
$$

This relation holds true for all $t$ such that $t_{n}+t \in\left[t_{n}, t_{n+1}\right]$, then we can at last prove the desired asymptotical estimate for $y$ :

$$
\left|y(t)-w_{k}(t-\theta)\right| \leq C e^{-\epsilon t}, \quad\left|\dot{y}(t)-\dot{w}_{k}(t-\theta)\right| \leq C e^{-\epsilon t} \quad \forall t>0 .
$$

So far we have focused our attention only on data of the form $\left(\eta\left(z_{0}, z_{1}\right), 0, z_{0}, z_{1}\right)$ belonging to the energy level $\mathcal{E}_{k}$, where $\left(z_{0}, z_{1}\right) \in \bar{B}_{\delta} \cap \mathcal{C}$. Actually every point of an orbit satisfying these initial condition leads to the same conclusion. Hence we can define the set of data for which the solution tends asymptotically to a pure flexural solution of given energy, putting together all the points belonging to orbits with initial data as above. 


\subsection{The case of system (1)}

Now we will turn our attention to the system (1), with hinged boundary conditions: if we suppose that the solutions are well approximated by their first mode of vibration, that is, after the rescaling,

$$
Y(x, t) \simeq y(t) \sin x, \quad \Theta(x, t) \simeq \theta(t) \sin x, \quad 0 \leq x \leq \pi .
$$

we have the following, more complicate, nonlinear system of ODE's:

$$
\begin{aligned}
& \ddot{y}(t)+\alpha y(t)+\psi_{1}(y, \theta)=0, \\
& \ddot{\theta}(t)+\beta \theta(t)+\gamma \psi_{2}(y, \theta)=0,
\end{aligned}
$$

where the nonlinear terms are given by the following formulas,

$$
\begin{gathered}
\psi_{1}(y, \theta)=\frac{2}{\pi} \int_{0}^{\pi}[f(y \sin x+l \sin (\theta \sin x))+f(y \sin x-l \sin (\theta \sin x))] \sin x d x, \\
\psi_{2}(y, \theta)=\frac{2}{\pi} \int_{0}^{\pi} l \cos (\theta \sin x)[f(y \sin x+l \sin (\theta \sin x))-f(y \sin x-l \sin (\theta \sin x))] \sin x d x .
\end{gathered}
$$

Let us define the non negative function

$$
\Phi(y, \theta)=\frac{2}{\pi} \int_{0}^{\pi}[F(y \sin x+l \sin (\theta \sin x))+F(y \sin x-l \sin (\theta \sin x))] d x .
$$

where $F(r)=\int_{0}^{r} f(s) d s$. We can immediately verify that

$$
\psi_{1}(y, \theta)=\frac{\partial \Phi}{\partial y}(y, \theta), \quad \psi_{2}(y, \theta)=\frac{\partial \Phi}{\partial \theta}(y, \theta),
$$

then also the system (24)-(25) admits a conserved energy, precisely

$$
\mathcal{E}(y, \dot{y}, \theta, \dot{\theta})=\frac{\dot{y}^{2}}{2}+\frac{\dot{\theta}^{2}}{2 \gamma}+\frac{\alpha}{2} y^{2}+\frac{\beta}{2 \gamma} \theta^{2}+\Phi(y, \theta) .
$$

We remark that $\psi_{1}(y, 0)=2 \tilde{f}(y)$, so that the pure flexural solution of (24)-(25), corresponding to the initial data $y(0)=k, \dot{y}(0)=0, \theta(0)=0, \theta \dot{(0)}=0$ is the same as the solution of (14), that is $y(t) \equiv w_{k}(t)$, and $\theta(t) \equiv 0$. The energy of such solution is, as expected, the energy $\mathcal{E}_{k}$ defined at the beginning of this section (let us note that $\Phi(y, 0)=2 \tilde{F}(y)$ ).

The construction and the properties of the Poincaré map around a pure flexural solution for the system (24)-(25) are the same as for the system (6)-(7). More important, the differential $\mathcal{L}_{k}$ of the Poincarè map at the origin is also the same! This is expected too, because the system (6)-(7) can be obtained from (24)-(25) linearizing with respect to $\theta, \dot{\theta}$ when they are supposed to be small. The proof of Proposition 4.1, and the entire Section 5 rely essentially on the properties of $\mathcal{L}_{k}$, then our main result holds without changes also for the system (24)-(25). Unfortunately this system is less suitable for providing numerical examples. 


\section{$5 \quad$ Asymptotic study of $\mathcal{L}_{k}$}

Let us assume for one moment that the function $\tilde{f}(r)$ is represented by the piecewise linear function $m r^{+}$(here $r^{+}$is the positive part of $r$ ). Strictly speaking this would not be admissible since it lacks differentiability in $r=0$, but the point is that, under assumption $(\tilde{\mathbf{H}})$, we will show that the asymptotic behavior of the Poincaré map as $k \rightarrow \infty$ is governed by this simple function. For such a function $\tilde{f}(r)$, it is an easy task to explicitly compute $w_{k}(t)$, the solution of the problem (14), $u(t)$ the solution of the equation (17), and the differential of the Poincaré map. In particular, we obtain that the period of $w_{k}(t)$ is independent of $k$ and is given by

$$
\tau_{\infty}=\frac{\pi}{\sqrt{\alpha}}+\frac{\pi}{\sqrt{\alpha+2 m}} .
$$

The interval $J_{\infty}=\left[0, \tau_{\infty}\right)$ is divided into three consecutive subintervals $I_{i}, i=1,2,3$, such that $w_{k}(t) \geq 0$ on $I_{1} \cup I_{3}, w_{k}(t) \leq 0$ on $I_{2}$. These intervals can be explicitly computed, precisely we have,

$$
I_{1}=\left[0, t_{0}\right), \quad I_{2}=\left[t_{0}, t_{0}+2 t_{1}\right), \quad I_{3}=\left[t_{0}+2 t_{1}, 2 t_{0}+2 t_{1}\right), \quad t_{0}=\frac{\pi}{2 \sqrt{\alpha+2 m}}, \quad t_{1}=\frac{\pi}{2 \sqrt{\alpha}} .
$$

It turns out that the time dependent coefficient of the Hill equation (17) is given by the piecewise constant $\tau_{\infty}$-periodic function:

$$
a_{\infty}(t)=\beta+2 m \gamma \quad \text { on } I_{1} \cup I_{3}, \quad a_{\infty}(t)=\beta \quad \text { on } I_{2} .
$$

Now we state the main result of this section which motivates the previous considerations:

Proposition 5.1 Assume that $(\tilde{\mathbf{H}})$ holds true. For $k>0$, let $u_{k}(t)$ be the solution of the Hill equation (17) with initial data

$$
u_{k}(0)=a, \quad \dot{u}_{k}(0)=b .
$$

Let $v$ be the solution of the problem

$$
\ddot{v}(t)+a_{\infty}(t) v(t)=0, \quad v(0)=a, \quad \dot{v}(0)=b,
$$

where $a_{\infty}$ is defined as in (28). Then for every $T>0$, we have

$$
\lim _{k \rightarrow \infty} u_{k}=v \text { in } C^{1}([0, T]) \text {. }
$$

The following convergence result for second order differential equations with a time-dependent coefficient will be essential in the proof of Proposition 5.1. It is special case of a classical theorem on the continuous parameter dependence for systems of linear differential equations [15]. For this reason we omit its proof.

Lemma 5.1 Let $T>0$, and suppose that the sequence of functions $\left(a_{k}(\cdot)\right)_{k \in \mathbb{N}}$ converges to $a_{\infty}(\cdot)$ in $L^{1}(0, T)$. Then the sequence $\left(u_{k}(\cdot)\right)_{k \in \mathbb{N}}$ of the solutions of the problems

$$
\ddot{u}_{k}(t)+a_{k}(t) u_{k}(t)=0, \quad u_{k}(0)=a, \quad \dot{u}_{k}(0)=b,
$$

converges in $C^{1}([0, T])$-norm to the solution $v(\cdot)$ of the limit problem:

$$
\ddot{v}(t)+a_{\infty}(t) v(t)=0, \quad v(0)=a, \quad \dot{v}(0)=b .
$$


PROOF OF PROPOSITION 5.1.

Let us consider the piecewise linear function $g(r)=m r^{+}$, and the solution $W(t)$ of the following initial value problem

$$
\ddot{W}(t)+\alpha W(t)+2 g(W(t))=0, \quad W(0)=1, \quad \dot{W}(0)=0 .
$$

For every $k>0$, let us denote by $a_{k}$ the coefficient of the Hill equation (17), that is

$$
a_{k}(t)=\beta+2 \gamma \tilde{f}^{\prime}\left(w_{k}(t)\right), \quad t \geq 0,
$$

where as usual $w_{k}$ is the solution of (14). The limit coefficient is given by the following formula (it is actually the same function defined in (28)),

$$
a_{\infty}(t)=\beta+2 \gamma g^{\prime}(W(t)) \quad t \geq 0
$$

From Lemma 5.1, it is clear that the assertion is proved if we show that for any given $T>0$,

$$
\lim _{k \rightarrow+\infty} a_{k}=a_{\infty}, \quad \text { in } \quad L^{1}([0, T]) .
$$

To start with, we claim that

$$
\lim _{k \rightarrow+\infty} \frac{\tilde{f}(k r)}{k}=g(r) \quad \text { uniformly on compact sets. }
$$

In order to prove the assertion (31) we fix any two positive numbers $R$ and $\delta$, with $0<\delta<R$, and denote by $I$ the closed interval $[-R, R]$. For every $r \in[-\delta, \delta]$, since $\tilde{f}(0)=0$, we have that

$$
\left|\frac{\tilde{f}(k r)}{k}-g(r)\right|=\left|\frac{\tilde{f}(k r)-\tilde{f}(0)}{k r} r-g(r)\right| \leq\left(\sup _{\mathbb{R}}\left|\tilde{f}^{\prime}\right|+m\right) \delta .
$$

On the other hand, thanks to condition $\tilde{b})$ in assumption $(\tilde{\mathbf{H}})$ and de l'Hôpital's rule, we have that $\lim _{x \rightarrow+\infty} \tilde{f}(x) / x=m$. Therefore for every $\varepsilon>0$, there exists $x_{\varepsilon}>0$ such that $|f(k r) / k r-m|<\varepsilon$, provided $k r>x_{\varepsilon}$. It follows that, if $k>x_{\varepsilon} / \delta$,

$$
\sup _{r \in[\delta, R]}\left|\frac{\tilde{f}(k r)}{k r} r-m r\right|<\varepsilon R .
$$

Thus, by definition of $g(r)$, and by simplification of $r$, we obtain

$$
\lim _{k \rightarrow+\infty}\left(\sup _{r \in[\delta, R]}\left|\frac{\tilde{f}(k r)}{k}-g(r)\right|\right)=0 .
$$

Similar considerations hold true in the interval $[-R,-\delta]$, hence

$$
\lim _{k \rightarrow+\infty}\left(\sup _{r \in I \backslash[-\delta, \delta]}\left|\frac{\tilde{f}(k r)}{k}-g(r)\right|\right)=0 .
$$


In conclusion, we have that

$$
\limsup _{k \rightarrow+\infty}\left\|\frac{\tilde{f}(k \cdot)}{k}-g(\cdot)\right\|_{L^{\infty}(I)} \leq\left(\sup _{\mathbb{R}}\left|\tilde{f}^{\prime}\right|+m\right) \delta
$$

which, by the arbitrariness of $\delta$, proves the assertion (31).

Now let us define the rescaled periodic function $W_{k}=w_{k} / k$, which clearly solves the following initial value problem,

$$
\ddot{W}_{k}(t)+\alpha W_{k}(t)+2 \frac{\tilde{f}\left(k W_{k}(t)\right)}{k}=0, \quad W_{k}(0)=1, \quad \dot{W}_{k}(0)=0 .
$$

Owing to the convergence result (31), and thanks to a classical continuous dependence theorem ([10] Thm. 3 Ch. XV, p. 297) we get that $W_{k}$ converges uniformly on $\mathbb{R}$ to $W$, the solution of problem (30).

As a consequence, thanks to the assumption $(\tilde{\mathbf{H}})$ and by definition of the function $g$, we get that for every $t \in[0, T]$ such that $W(t) \neq 0$,

$$
\lim _{k \rightarrow+\infty} \tilde{f}^{\prime}\left(w_{k}(t)\right)=\lim _{k \rightarrow+\infty} \tilde{f}^{\prime}\left(k W_{k}(t)\right)=g^{\prime}(W(t)),
$$

hence the sequence $\left(a_{k}\right)$ converges almost everywhere on $[0, T]$ to the function $a_{\infty}$. Since $\tilde{f}^{\prime}$ and $g^{\prime}$ are both bounded, the dominated convergence theorem yields convergence in $L^{1}([0, T])$. The conclusion follows from Lemma 5.1 and the definition of $u_{k}, w_{k}, v$ and $W$.

To conclude the proof of our main result we need to know that the sequence of periods of $w_{k}$ converges to the limit period $\tau_{\infty}$. This is indeed the case for any function $\tilde{f}$ satisfying $(\tilde{\mathbf{H}})$, as we prove in the following Proposition.

Proposition 5.2 Let $w_{k}(t)$ be the solution of the problem (14) in which $f$ satisfies assumption $(\tilde{\mathbf{H}})$, and let $\tau_{k}$ be its period. Then $\tau_{k} \rightarrow \tau_{\infty}$, where $\tau_{\infty}$ has been defined in (26).

PROOF. The interval $J_{k}=\left[0, \tau_{k}\right)$ is divided into three subintervals, $J_{k}=I_{1, k} \cup I_{2, k} \cup I_{3, k}$, such that $w_{k}(t) \geq 0$ on $I_{1, k} \cup I_{3, k}, w_{k}(t) \leq 0$ on $I_{2, k}$. We are going to prove that

$$
\lim _{k \rightarrow \infty}\left|I_{1, k}\right|=\lim _{k \rightarrow \infty}\left|I_{3, k}\right|=t_{0}, \quad \lim _{k \rightarrow \infty}\left|I_{2, k}\right|=2 t_{1},
$$

where $|I|$ stands for the amplitude of the interval $I$.

Since for every $t \in \mathbb{R}$ the energy of $w_{k}$ is conserved, that is

$$
\frac{1}{2} \dot{w}_{k}(t)^{2}+\frac{\alpha}{2} w_{k}(t)^{2}+2 \tilde{F}\left(w_{k}(t)\right)=\mathcal{E}_{k},
$$

in the first interval $I_{1, k}$ in which $w_{k}$ is positive and $\dot{w}_{k}$ is negative, we may write

$$
\dot{w}_{k}(t)=-\sqrt{2 \mathcal{E}_{k}-\alpha w_{k}(t)^{2}-4 \tilde{F}\left(w_{k}(t)\right)}, \quad t \in I_{1, k} .
$$

Thus the length $t_{0}(k)$ of $I_{1, k}$ is given by the following integral,

$$
t_{0}(k)=\int_{0}^{k} \frac{d r}{\sqrt{2 \mathcal{E}_{k}-\alpha r^{2}-4 \tilde{F}(r)}}=\int_{0}^{1} \frac{d r}{\sqrt{2 \mathcal{E}_{k} / k^{2}-\alpha r^{2}-4 \tilde{F}(k r) / k^{2}}} .
$$


By recalling the initial data for the problem (14), the expression under square root is explicitly given by

$$
2 \mathcal{E}_{k} / k^{2}-\alpha r^{2}-4 \tilde{F}(k r) / k^{2}=\alpha+4 \tilde{F}(k) / k^{2}-\alpha r^{2}-4 \tilde{F}(k r) / k^{2} .
$$

Thanks to the assumption $(\tilde{\mathbf{H}})$, and by an application of de l'Hôpital's rule, for every $r>0$ we have that

$$
\lim _{k \rightarrow+\infty} \tilde{F}(k r) / k^{2}=m r^{2} / 2,
$$

thus

$$
\lim _{k \rightarrow+\infty}\left(2 \mathcal{E}_{k} / k^{2}-\alpha r^{2}-4 \tilde{F}(k r) / k^{2}\right)=(\alpha+2 m)\left(1-r^{2}\right), \quad r>0 .
$$

Since $\tilde{F}$ is increasing on the interval of positive numbers, we have $\tilde{F}(k)-\tilde{F}(k r) \geq 0$ for every $r \in[0,1]$ and $k>0$, so that the integrand in (33) is dominated by $1 / \sqrt{\alpha-\alpha r^{2}}$. Passing to the limit as $k \rightarrow+\infty$, thanks to the dominated convergence theorem, we obtain

$$
\lim _{k \rightarrow+\infty} t_{0}(k)=\frac{1}{\sqrt{\alpha+2 m}} \int_{0}^{1} \frac{d r}{\sqrt{1-r^{2}}}=\frac{\pi}{2 \sqrt{\alpha+2 m}} .
$$

Let us now consider the interval in which both $w_{k}$ and $\dot{w}_{k}$ are negative. The same equation (32) as in the previous case is satisfied, and if we call by $s_{k}$ the unique negative number such that

$$
\frac{\alpha}{2} s_{k}^{2}+2 \tilde{F}\left(s_{k}\right)=\mathcal{E}_{k},
$$

we have that half of the length of $I_{2, k}$ is given by

$$
t_{1}(k)=\int_{s_{k}}^{0} \frac{d r}{\sqrt{2 \mathcal{E}_{k}-\alpha r^{2}-4 \tilde{F}(r)}}=\int_{0}^{1} \frac{d r}{\sqrt{2 \mathcal{E}_{k} / s_{k}^{2}-\alpha r^{2}-4 \tilde{F}\left(s_{k} r\right) / s_{k}^{2}}},
$$

Note that the expression under square root in this case may be written as follows

$$
2 \mathcal{E}_{k} / s_{k}^{2}-\alpha r^{2}-4 \tilde{F}\left(s_{k} r\right) / s_{k}^{2}=\alpha-\alpha r^{2}+4 \tilde{F}\left(s_{k}\right) / s_{k}^{2}-4 \tilde{F}\left(s_{k} r\right) / s_{k}^{2},
$$

and that, since $\lim _{k \rightarrow+\infty} s_{k}=-\infty$,

$$
\lim _{k \rightarrow+\infty} \tilde{F}\left(s_{k} r\right) / s_{k}^{2}=0, \quad r>0 .
$$

Arguing as before, we get $\lim _{k \rightarrow+\infty} t_{1}(k)=\pi /(2 \sqrt{\alpha})$. The remaining interval $I_{3, k}$ in which $w_{k}$ is positive has obviously the same length of $I_{1, k}$.

\subsection{Proof of Theorem 1}

From Proposition 4.1 we know that if the equal diagonal entries of the matrix $\mathcal{L}_{k}$ are greater than 1 in modulus, then the periodic pure flexural solution $w_{k}$ is unstable. In particular, we remind that $L_{11}=u_{k}\left(\tau_{k}\right)$, where $u_{k}$ is the solution of (17) with initial data $u_{k}(0)=1, \dot{u}_{k}=0$. 
From Proposition 5.1, we have that $u_{k} \rightarrow v$ uniformly on every bounded interval, where $v$ is the solution of $(29)$ with data $v(0)=1, \dot{v}(0)=0$, and from Proposition 5.2, we know that $\tau_{k} \rightarrow \tau_{\infty}$. As a consequence, we get that

$$
\lim _{k \rightarrow+\infty} u_{k}\left(\tau_{k}\right)=v\left(\tau_{\infty}\right)
$$

A straightforward computation (see below) shows that

$$
v\left(\tau_{\infty}\right)=\cos \phi_{0} \cos \phi_{1}-\frac{q+q^{-1}}{2} \sin \phi_{0} \sin \phi_{1},
$$

in which $\phi_{0}, \phi_{1}$ are defined in (10). Therefore, if the condition on the parameters of Theorem 1 is satisfied, and if the energy, or equivalently the positive number $k$, is sufficiently large, the pure flexural solution is unstable.

We conclude by outlining the main steps of the computation leading to (34). First let us rename conveniently the constants in (28):

$$
a_{\infty}(t)=\beta+2 m \gamma=A^{2} \quad \text { on } I_{1} \cup I_{3}, \quad a_{\infty}(t)=\beta=B^{2} \quad \text { on } I_{2} .
$$

Obviously we have $v(t)=\cos (A t), \dot{v}(t)=-A \sin (A t)$ for $t \in I_{1}$. Therefore we may calculate $v$ and $\dot{v}$ on $I_{2}$ by using $v\left(t_{0}\right)=\cos \left(A t_{0}\right)$ and $\dot{v}\left(t_{0}\right)=-A \sin \left(A t_{0}\right)$ as initial data, and at the end of $I_{2}$ we get

$$
\begin{gathered}
v\left(t_{0}+2 t_{1}\right)=\cos \left(A t_{0}\right) \cos \left(2 B t_{1}\right)-\frac{A}{B} \sin \left(A t_{0}\right) \sin \left(2 B t_{1}\right), \\
\dot{v}\left(t_{0}+2 t_{1}\right)=-B \cos \left(A t_{0}\right) \sin \left(2 B t_{1}\right)-A \sin \left(A t_{0}\right) \cos \left(2 B t_{1}\right) .
\end{gathered}
$$

Finally we compute $v$ on $I_{3}$ using the last expressions for $v\left(t_{0}+2 t_{1}\right), \dot{v}\left(t_{0}+2 t_{1}\right)$ as initial data. At the end of $I_{3}$ we have:

$$
\begin{aligned}
v\left(t_{\infty}\right)= & v\left(2 t_{0}+2 t_{1}\right)=\left(\cos \left(A t_{0}\right) \cos \left(2 B t_{1}\right)-\frac{A}{B} \sin \left(A t_{0}\right) \sin \left(2 B t_{1}\right)\right) \cos \left(A t_{0}\right)- \\
- & \frac{1}{A}\left(B \cos \left(A t_{0}\right) \sin \left(2 B t_{1}\right)+A \sin \left(A t_{0}\right) \cos \left(2 B t_{1}\right)\right) \sin \left(A t_{0}\right)= \\
= & \cos \left(2 A t_{0}\right) \cos \left(2 B t_{1}\right)-\frac{1}{2}\left(\frac{A}{B}+\frac{B}{A}\right)\left(\sin \left(2 A t_{0}\right) \sin \left(2 B t_{1}\right) .\right.
\end{aligned}
$$

By the definitions of $t_{0}, t_{1}$ in (27), $A, B$ in (35), and $\phi_{0}, \phi_{1}$ in (10) we have $2 A t_{0}=\phi_{0}$, $2 B t_{1}=\phi_{1}, q=\frac{A}{B}$.

\section{References}

[1] G. Arioli, F. Gazzola, A new mathematical explanation of what triggered the catastrophic torsional mode of the Tacoma Narrows Bridge, Appl. Math. Model. 39 (2015), no. 2, 901912

[2] E. Berchio, F. Gazzola, A qualitative explanation of the origin of torsional instability in suspension bridges, Nonlinear Anal. 121 (2015), 5472

[3] T. Cazenave , F.B. Weissler, Unstable simple modes of the nonlinear string, Quart. Appl. Math. 54 (1996), no. 2. 287-305 
[4] S.R. Deans, Radon and Abel Transforms, in The Transforms and Applications Handbook: Second Edition. Ed. Alexander D. Poularikas Boca Raton: CRC Press LLC, 2000

[5] P. Drábek, H. Leinfelder, G. Tajčová, Coupled string-beam equations as a model of suspension bridges, Appl. Math., 44 (1999), pp. 97142

[6] F. Gazzola, Mathematical Models for Suspension Bridges: Nonlinear Structural Instability, MS\&A. Modeling, Simulation and Applications, 15. Springer, Cham, 2015

[7] M. Ghisi, M. Gobbino, Unstable simple modes for a class of Kirchhoff equations. Ann. Fac. Sci. Toulouse Math. (6) 10 (2001), no. 4, 639658

[8] J. Glover, A. C. Lazer, P. J. McKenna, Existence and stability of large scale nonlinear oscillations in suspension bridges Z. Angew. Math. Phys. 40 (1989), no. 2, 172200

[9] P. Hartman, Ordinary differential equations. John Wiley \& Sons, Inc., New York-LondonSydney 1964

[10] M.W. Hirsch, S. Smale, Differential equations, dynamical systems, and linear algebra. Pure and Applied Mathematics, Vol. 60. Academic Press, New York-London, 1974

[11] A. C. Lazer, P. J. McKenna, Large-amplitude periodic oscillations in suspension bridges: some new connections with nonlinear analysis, SIAM Rev., 32 (1990), 537578

[12] P.J. McKenna, W. Walter, Nonlinear oscillations in a suspension bridge, Arch. Ration. Mech. Anal., 98 (1987), 167177

[13] P. J. McKenna, C. Ó Tuama, Large torsional oscillations in suspension bridges visited again: vertical forcing creates torsional response, Amer. Math. Monthly 108 (2001)

[14] K. S. Moore, Large torsional oscillations in a suspension bridge: multiple periodic solutions to a nonlinear wave equation, SIAM J. Math. Anal. 33 (2002), no. 6, 14111429

[15] Z. Opial, Continuous parameter dependence in linear systems of differential equations, J. Differential Equations 3 (1967), 571579 